Supporting Information for:

\title{
Nitrogen-Doping Through Two-Step Pyrolysis of Polyacrylonitrile on Graphite Felts for Vanadium Redox Flow Batteries
}

Sang Jun Yoon, ${ }^{*} \dagger, \ddagger$ Sangwon Kim,${ }^{\S}$ Dong Kyu Kim,,$~ D u k$ Man Yu, ${ }^{\dagger}$ Rolf Hempelmann, ${ }^{\ddagger}$ Young Taik Hong, ${ }^{\dagger}$ Soonyong So ${ }^{*} \dagger$

${ }^{\dagger}$ Energy Materials Research Center, Korea Research Institute of Chemical Technology (KRICT), Daejeon 34114, South Korea

¥Transfercentre Sustainable Electrochemistry, Saarland University, Saarbrücken 66123, Germany

${ }^{\S}$ Bio Sensor Group, Korea Institute of Science and Technology Europe (KIST-EU), Saarbrücken 66123, Germany

"School of Mechanical Engineering, Chung-Ang University, Seoul 06974, South Korea

\section{AUTHOR INFORMATION \\ Corresponding Authors}

Sang Jun Yoon

*Email: sjyoon@ krict.re.kr (S.J.Y).

Soonyong So

*Email: syso@ krict.re.kr (S.S). 


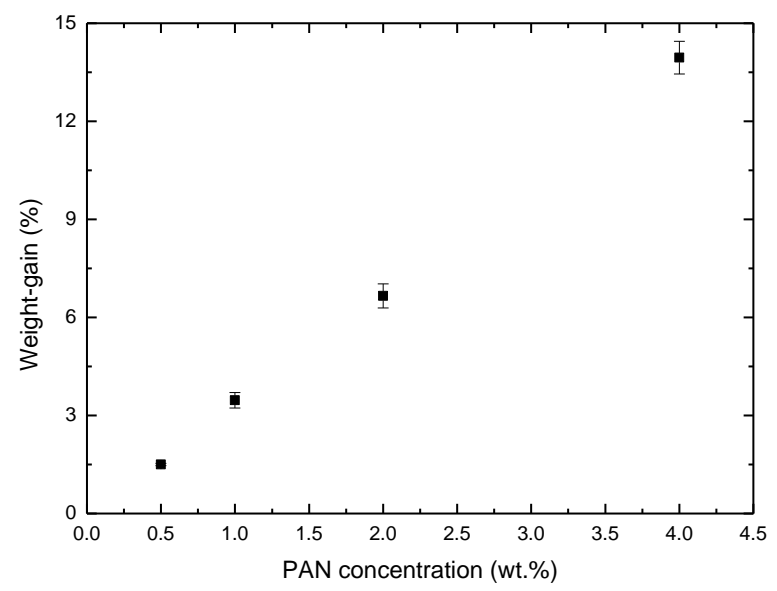

Figure S1. Weight-gain of electrodes depending on the PAN concentration. The weight-gain $(m=$ $\left.\left(w_{\mathrm{p}}-w_{0}\right) / w_{0} \times 100(\%)\right)$ was evaluated by the weights of pristine electrode $\left(w_{0}\right)$, and an electrode after two-step pyrolysis $\left(w_{\mathrm{p}}\right)$.
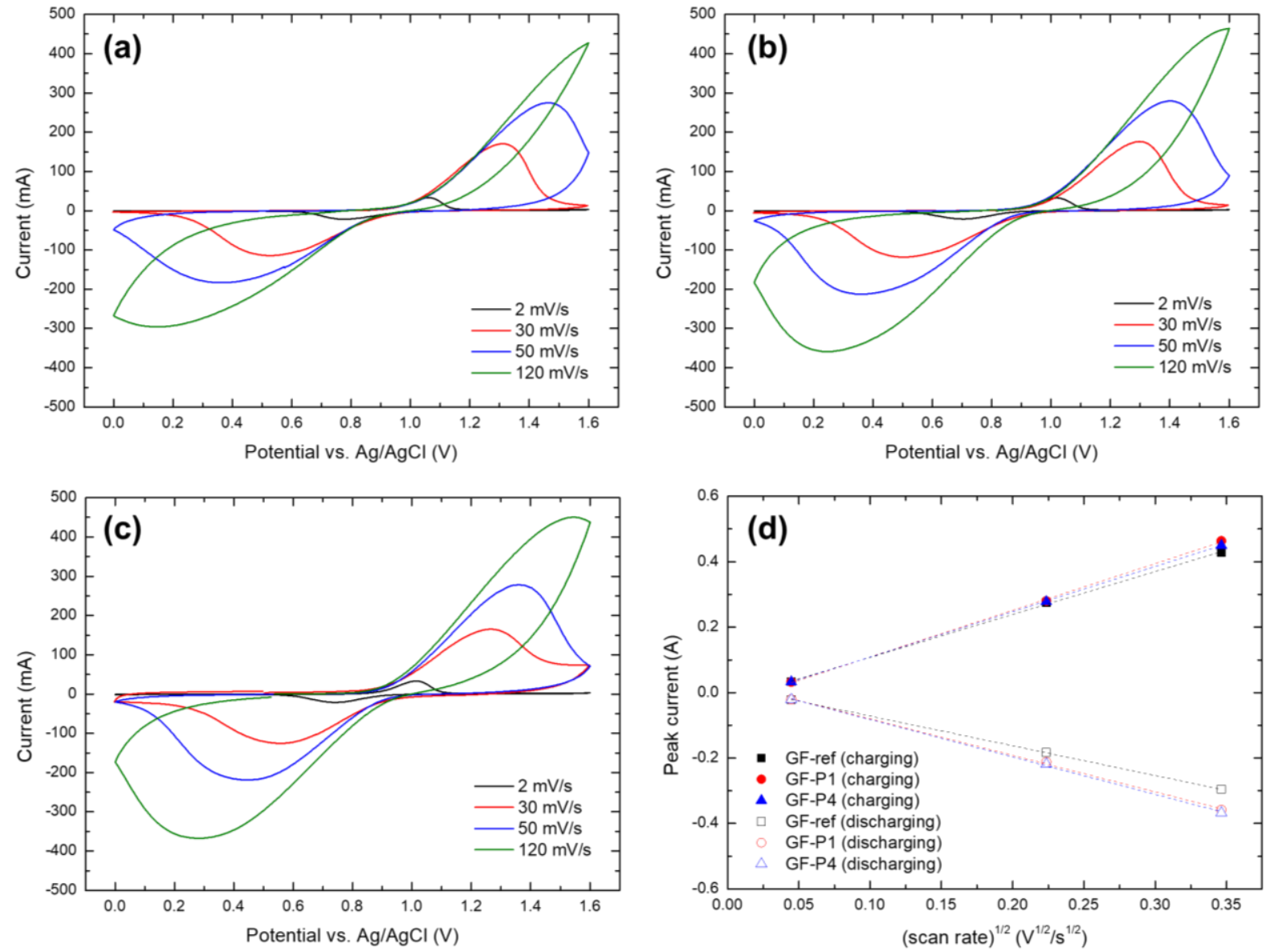

Figure S2. Cyclic voltammograms of (a) GF-ref, (b) GF-P1, and (c) GF-P4 in $0.05 \mathrm{M} \mathrm{VOSO}_{4}$ in $3.0 \mathrm{M} \mathrm{H}_{2} \mathrm{SO}_{4}$ electrolyte at a scan rate of 2, 30, 50, and $120 \mathrm{mV} / \mathrm{s}$. (d) The peak current versus the square root of scan rates. 
(a)

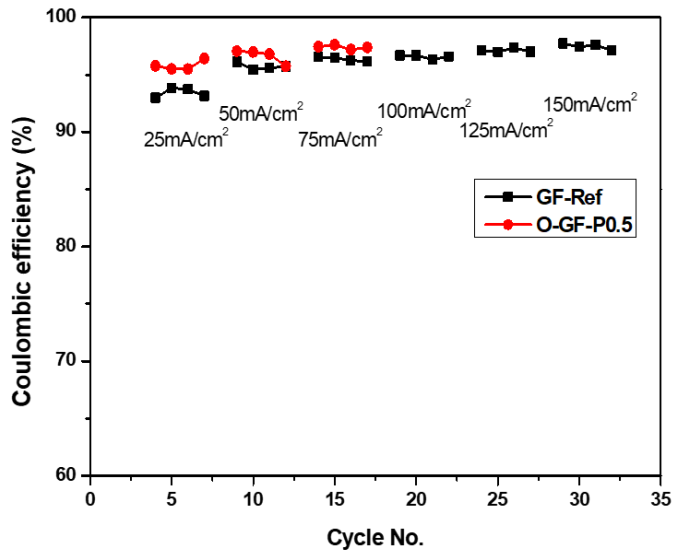

(b)

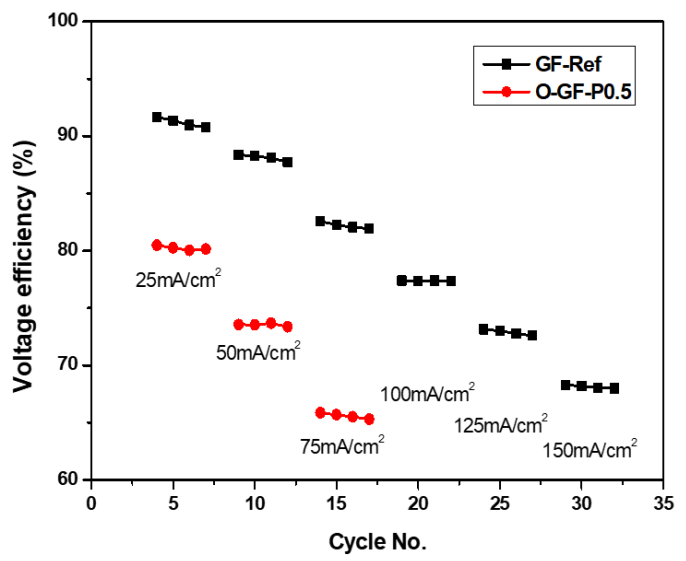

(c)

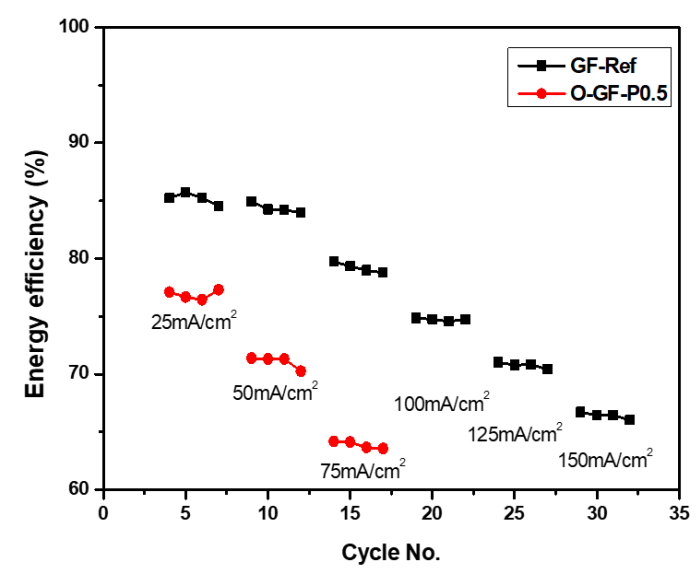

Figure S3. Cycling performance of VRFBs employing GF-ref and O-GF-P0.5 at different current densities; (a) Coulombic efficiency, (b) voltage efficiency, and (c) energy efficiency. 
(a)

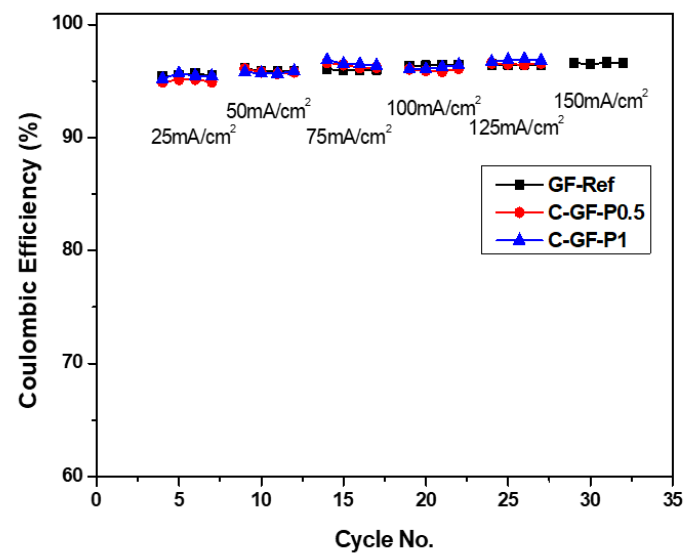

(b)

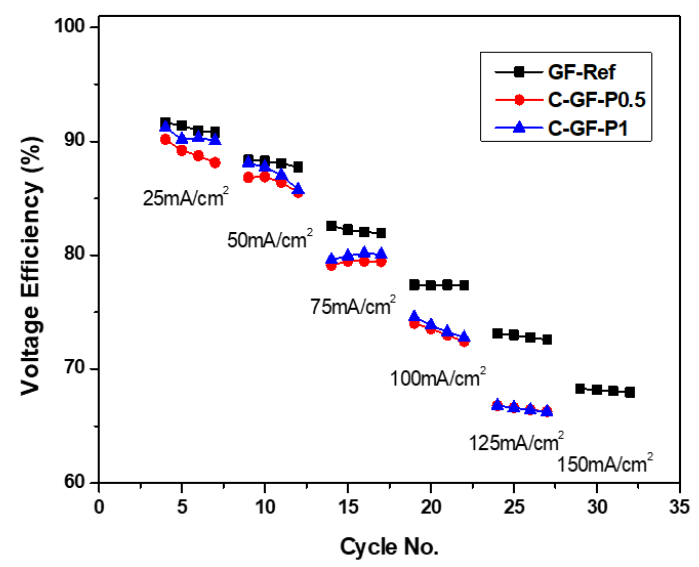

(c)

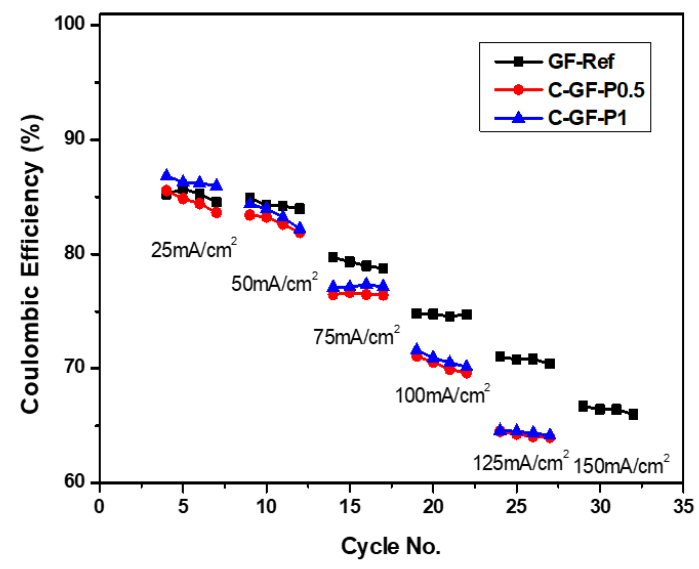

Figure S4. Cycling performance of VRFBs employing GF-ref, C-GF-P0.5 and C-GF-P1 at different current densities; (a) Coulombic efficiency, (b) voltage efficiency, and (c) energy efficiency. 

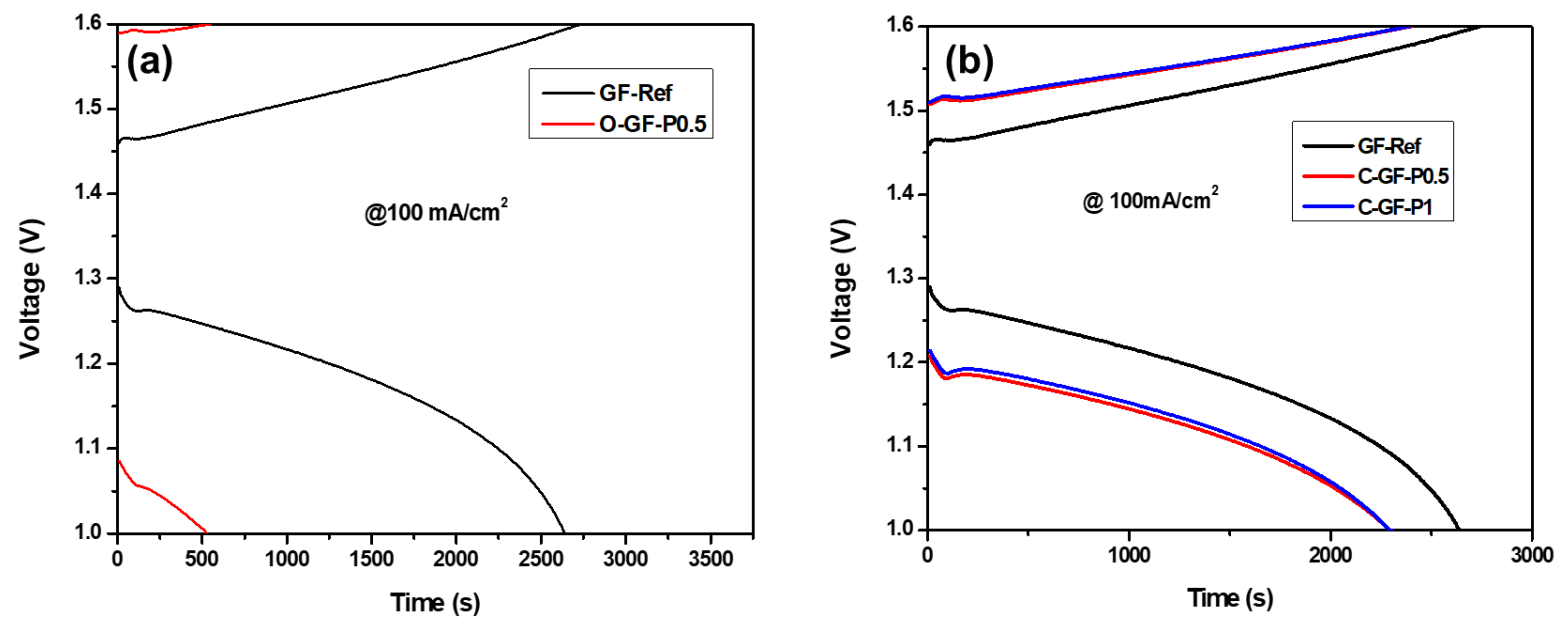

Figure S5. Charge/discharge curves for VRFBs employing (a) GF-ref and O-GF-P0.5, and (b) GF-ref, C-GF-P0.5 and C-GF-P1 at the current density of $100 \mathrm{~mA} / \mathrm{cm}^{2}$. 